\title{
Feynman Propagator for Closed Timelike Curves in the Kerr Metric
}

\author{
Miguel Socolovsky \\ Instituto de Ciencias Nucleares, Universidad Nacional Autónoma de México, Cd. Universitaria, 04510, Ciudad \\ de México, México \\ Email: socolovs@nucleares . unam.mx
}

\begin{abstract}
We compute the Feynman propagator associated with closed timelike curves in the neighborhood of the ring singularity in the Kerr metric. The propagator is well defined outside $r=0$, where it ceases to exist.
\end{abstract}

Keywords: Kerr metric, closed timelike curves, Feynman propagators.

\section{Introduction}

The Penrose-Carter diagram [1] of the Kruskal-Szekeres [2,3] maximal analytic extension of the Kerr solution [4] to Einstein's equations, exhibits a quadruple-infinity of asymptotically flat regions: ..., $\mathcal{U}_{-1}$, $\mathcal{U}_{0}, \mathcal{U}_{1}, \ldots$ (anyone can be our "initial" universe), .., $\overline{\mathcal{U}}_{-1}, \overline{\mathcal{U}}_{0}, \overline{\mathcal{U}}_{1} \ldots$ (mirror images of the $\mathcal{U}_{k}^{\prime} s$ ), and $\ldots, \mathcal{U}_{-1}^{\prime}, \mathcal{U}_{0}^{\prime}, \mathcal{U}_{1}^{\prime}, \ldots$ and their mirror images $\ldots, \overline{\mathcal{U}}_{-1}^{\prime}, \overline{\mathcal{U}}_{0}^{\prime}, \overline{\mathcal{U}}_{1}^{\prime} \ldots$ To the last two families correspond a negative value of the $r$ coordinate which, as can be easily seen through the Newman-Janis algorithm [5], is analytically continued from a polar coordinate $r>0$ to a Cartesian one with range $(-\infty,+\infty)$. It is interesting that any future directed timelike trajectory can start at any spacetime point in, say, $\mathcal{U}_{k}$, and end at any point in, say, $\mathcal{U}_{l}^{\prime}[6]$, after passing through many black hole (BH) and white hole (WH) regions, and through an open disk whose boundary is one of the infinite copies of the ring singularity [7]. The important point is that $\mathcal{U}_{l}^{\prime} \neq \mathcal{U}_{k}$ for all $l, k$, with $\mathcal{U}_{l}^{\prime}$ and $\mathcal{U}_{k}$ both Minkowskian respectively at $r=-\infty$ and $r=+\infty$. (The sets $\mathcal{U}_{k}$ 's and $\mathcal{U}_{k}^{\prime}$ 's together with their mirror images are respectively denoted by I and III in Fig. 3 of Ref. [1], while the BH and WH regions are denoted by II.)

If one requires that the physical solution (possibly the final stage of the gravitational collapse of a spinning star) be globally hyperbolic, which is the strongest sufficient condition for the absence of closed causal curves and therefore for causality protection [8], the only surviving region is any of the central "diamond" shaped portions of the diagram, given by the union $\mathcal{U}_{k} \cup \overline{\mathcal{U}}_{k} \cup B H_{k} \cup W H_{k}$, with upper and lower boundaries the Cauchy horizons of a Cauchy surface $S$ through the center of the diamond, future and past event horizons and null infinities. (The analogue diamond for the Reissner-Nördstrom case can be seen in Fig. 13 of Ref. [8].) In $B H_{k}$ and $W H_{k}$, the vectors $\partial_{r}$ and $\partial_{t}$ are respectively timelike and spacelike, and the constant $r$ lines decrease from $r_{+}$to $r_{-}$in $B H_{k}$ and increase from $r_{-}$to $r_{+}$in $W H_{k}$. The asymptotically flat region $\mathcal{U}_{k}^{\prime} \cup \overline{\mathcal{U}}_{k}^{\prime}$ which extends from $r_{-}$to $r=-\infty$ and includes the left and right timelike singularities at $r=0$, remains outside the physical solution. This does not occur in the Schwarzschild case, since from the outset the solution is globally hyperbolic.

Nevertheless, in Section 2, we shall consider the maximal analytic extension of the Kerr solution mentioned at the beginning, which is not globally hyperbolic, and compute the Feynman propagators associated with the causality violating closed timelike curves which exist in the neighborhood of the singularity rings. The propagators exist and are well defined in these regions but, as expected, cease to exist at the singularities due to the infinite oscillations of imaginary exponential functions. Section 3 is devoted to conclusions.

\section{Metric, Lagrangian, and Propagator}

In Boyer-Lindquist [9] coordinates $x^{\mu}=\left(x^{0}, x^{1}, x^{2}, x^{3}\right)=(t, r, \theta, \psi)$, at $t=t_{0}=$ const., $\theta=\pi / 2$ (equator), $r$ negative but close to the singularity ring at $r=0$, for the cases $a^{2}<M^{2}$ and $a=M$ 
(extreme Kerr), the Kerr metric is approximately given by (we use the metric signature $(+,-,-,-)$ )

$$
d s^{2}=g_{\psi \psi} d \psi^{2}=\frac{2 M a^{2}}{|r|} d \psi^{2} .
$$

So, the infinitesimal spacetime interval is timelike and, since $\psi \in[0,2 \pi]$, one has a closed timelike curve as $\psi$ goes from 0 to $2 \pi$. $M$ is the mass of the black hole and $a$ is the angular momentum/unit mass and also the radius of the singularity ring. With $\psi=\psi(\lambda), \lambda \in[0, \Lambda]$ is a parameter with units of lenght, $\Lambda$ being a lenght scale.

The interval (1) allows to define a dimensionless Lagrangian density

$$
\mathcal{L}(\psi, \dot{\psi} ; \lambda)=\left(\frac{d s}{d \lambda}\right)^{2}=\frac{1}{2} \mu \dot{\psi}^{2}
$$

("free particle" Lagrangian) where $\mu=\frac{4 M a^{2}}{|r|}$ and $\dot{\psi}=u^{\psi}=\frac{d \psi}{d \lambda}$. The Lagrange equation $\frac{d}{d \lambda}\left(\frac{\partial \mathcal{L}}{\partial \dot{\psi}}\right)=\frac{\partial \mathcal{L}}{\partial \psi}$ leads to $\mu \ddot{\psi}=0$ with solution

$$
\bar{\psi}(\lambda)=A \lambda+B .
$$

With $\bar{\psi}(0)=B=0$ and $\bar{\psi}(\Lambda)=A \Lambda=2 \pi$ one has the solution

$$
\bar{\psi}(\lambda)=\frac{2 \pi}{\Lambda} \lambda
$$

with a classical action

$$
\bar{S}=\frac{1}{\Lambda} \int_{0}^{\Lambda} d \lambda \mathcal{L}(\bar{\psi}, \dot{\bar{\psi}} ; \lambda)
$$

given by $8 \pi^{2} M a^{2} /|r| \Lambda^{2}$.

It is immediate to ask oneself which quantum object, if any, could be associated to the above closed timelike path. A natural (and almost unique) is the Feynman propagator $K_{\Lambda}(2 \pi, \Lambda ; 0,0)$ given by the path integral

$$
\int_{\psi(0)=0}^{\psi(\Lambda)=2 \pi} \mathcal{D} \psi e^{i / \Lambda \int_{0}^{\Lambda} d \lambda \mathcal{L}(\psi, \dot{\psi} ; \lambda)}=\int_{\psi(0)=0}^{\psi(1)=2 \pi} \mathcal{D} \psi e^{i \int_{0}^{1} d \sigma \mathcal{L}\left(\psi, \psi^{\prime} ; \sigma\right)}=K_{\Lambda}(2 \pi, 1 ; 0,0),
$$

where $\sigma$ is the dimensionless parameter $\frac{\lambda}{\Lambda} \in[0,1]$ and $\psi^{\prime}=\frac{d \psi}{d \sigma}$. The path integral is that corresponding to a "particle" of mass $m=\frac{\mu}{\Lambda^{2}}$ moving along a ring of unit radius. The result is well known and is given by $[10]$

$$
K_{\Lambda}(2 \pi, 1 ; 0,0)=\frac{a}{\Lambda} \sqrt{\frac{2 M}{\pi|r|}} \sum_{l=-\infty}^{l=+\infty} \exp \left(i\left(\frac{8 M}{|r|}(\pi a / \Lambda)^{2}(1-l)^{2}-\pi / 4\right)\right),
$$

which has units of $(\text { lenght })^{0}$ and is well defined in the range $0<|r| \ll a \leq M . K_{\Lambda}$ involves the Jacobi theta function $[11]$

$$
\Theta_{3}(z, t)=\sum_{l=-\infty}^{l=+\infty} e^{i\left(\pi t l^{2}+2 l z\right)}
$$

with $t=\frac{8 \pi M a^{2}}{|r| \Lambda^{2}}$ and $z=-\pi t . l$ is the winding number and corresponds to a classical path contributing to the path integral giving $l$ additional turns to the ring as the particle completes a circle. The $l=0$ contribution corresponds to the free particle case.

With $a$ and $M$ at our disposal, for $M>a$ there are two natural choices for the lenght scale $\Lambda$ :

$$
\Lambda_{1}=\frac{a^{2}}{M}<a \quad \text { and } \quad \Lambda_{2}=\frac{M^{2}}{a}>M .
$$

These choices give the propagators

$$
K_{\Lambda_{1}}(2 \pi, 1 ; 0,0)=\frac{M}{a} \sqrt{\frac{2 M}{\pi|r|}} \sum_{l=-\infty}^{l=+\infty} \exp \left(i\left(\frac{8 M}{|r|}(\pi M / a)^{2}(1-l)^{2}-\pi / 4\right)\right),
$$


and

$$
K_{\Lambda_{2}}(2 \pi, 1 ; 0,0)=\left(\frac{a}{M}\right)^{2} \sqrt{\frac{2 M}{\pi|r|}} \sum_{l=-\infty}^{l=+\infty} \exp \left(i\left(\frac{8 M}{|r|} \pi^{2}(a / M)^{4}(1-l)^{2}-\pi / 4\right)\right) .
$$

For the extreme Kerr case, $\Lambda_{1}=\Lambda_{2}=M=a$, the propagator is

$$
K_{\Lambda=M=a}(2 \pi, 1 ; 0,0)=M \sqrt{\frac{2 M}{\pi|r|}} \sum_{l=-\infty}^{l=+\infty} \exp \left(i\left(\frac{8 \pi^{2} M}{|r|}(1-l)^{2}-\pi / 4\right)\right) .
$$

In all cases, it is clear that $K_{\Lambda}$ does not exist in the $r \rightarrow 0_{-}$limit (the singularity), because of the infinite oscillations of the exponentials in (7).

\section{Conclusion}

We conclude that, though at the present time the assignation of a quantum character to the Feynman propagator associated to a closed timelike curve and in general to an arbitrary timelike or null geodesic [12] can only be considered a formal assignation, it nevertheless suggests that even a complete quantum treatment of the Kerr black hole would not eliminate the presence of such "time machines", which, however, remain hidden behind the event and Cauchy horizons of any starting universe $\mathcal{U}_{k}$ and, moreover, outside any globally hyperbolic portion of the solution. Finally, we want to emphasize that locally, any displacement along a closed timelike (or null) curve is always forward in "time"; the time machine effect only emerges globally, due to the warping of the spacetime.

\section{References}

1. Carter, B. Complete Analytic Extension of the Symmetry Axis of Kerr's Solution of Einstein's Equations, Phys. Rev. 141, 1242-1247 (1966).

2. Kruskal, M.D. Maximal extension of Schwarzschild metric, Phys. Rev. 119, 1743-1745 (1960).

3. Szekeres, G. On the singularities of a Riemannian manifold, Publ. Math. Debrecen 7, 285-301 (1960).

4. Kerr, R.P. Gravitational Field of a Spinning Mass as an Example of Algebraically Special Metrics, Phys. Rev. Lett. 11, 237-238 (1963).

5. Newman, E.T. and Janis, A.I. Note on the Kerr Spinning-Particle Metric, J. Math. Phys. 6, 915-917 (1965).

6. Battistotti, G. Closed Timelike Curves in the Kerr Metric, Thesis Laurea, Università degli Studi di Trento (2014/2015).

7. Carroll, S. Spacetime and Geometry. An Introduction to General Relativity, p. 265, Addison-Wesley, San Francisco (2004).

8. Witten, E. Light rays, singularities, and all that, Rev. Mod. Phys. 92, 045004, 1-49 (2020)

9. Boyer, R.H. and Lindquist, R.W. Maximal Analytic Extension of the Kerr Metric, J. Math. Phys 8, 265-281 (1967).

10. Ingold, G-L. Path Integrals and Their Application to Dissipative Quantum Systems, in Coherent Evolution in Noisy Enviroments, Lecture Notes Phys. 611, p. 9, Springer (2002).

11. Schulman, L.S. Techniques and Applications of Path Integration, p. 193, Wiley (1981).

12. Socolovsky, M. Quantum propagators for geodesic congruences, Theoretical Physics 6, Number 2, 9-17 (2021). 